\title{
A Parallel Evolutionary Algorithm for Unconstrained Binary Quadratic Problems
}

\author{
István Borgulya \\ Faculty of Business and Economics \\ University of Pécs, Hungary \\ Tel.: 3672501599 \\ borgulya@ktk.pte.hu
}

\begin{abstract}
In this paper an island model is described for the unconstrained Binary Quadratic Problem (BQP), which can be used with up to 2500 binary variables. Our island model uses a master-slave structure and the migration is centralized. In the model a basic evolutionary algorithm (EA) runs which is a hybrid, steady-state EA. The basic EA uses a new mutation operator that is composed of two parts and based on a modified version of an explicit collective memory method (EC-memory), the Virtual Loser [2].We tested our island model on the benchmark problems from the OR-Library. Comparing the results with other heuristic methods, we can conclude that our algorithm is highly effective in solving large instances of the BQP; it has a high probability of finding the best-known solutions.
\end{abstract}

\section{Categories and Subject Descriptors}

12.8 [Artificial Intelligence]: Problem Solving, Control Methods and Search - heuristic methods

\section{General Terms}

Algorithms.

\section{Keywords}

Binary quadratic programming; evolutionary algorithm; island model; EC-memory.

\section{INTRODUCTION}

In the BQP, a symmetric $n \times n$ matrix $Q=\left(q_{i j}\right)$ is given, and a binary vector $X$ of length $n$ is desired, which maximizes the objective function

$$
f(X)=X^{2} Q X=\sum_{i=1}^{n} \sum_{i=1}^{n} q_{t j} X_{t} X f_{t} \quad X_{t} \in\{0,1\} \forall t
$$

$\mathrm{BQP}$ has a central role in combinatorial optimization. A large number of problems can be formulated as maximization of quadratic real values function in 0-1 variables.

In this paper, we present now a new EA for the BQP. First we develop a memetic algorithm, a "basic EA", next we organize a parallel, island model with the help of the basic EA. The basic algorithm is a hybrid, steady-state EA. It uses a truncation selection, a new mutation operation, and the used local search

Copyright is held by the author/owner(s).

GECCO'08, July 12-16, 2008, Atlanta, Georgia, USA.

ACM 978-1-60558-130-9/08/07. method is the randomized k-opt procedure. The mutation operation is composed of two parts and based on a new modified version of the Virtual Loser method.

\section{THE ALGORITHM}

The main attributes of the basic EA are the following:

- The basic EA uses 2-stage algorithm structure. The first stage is a quick "preparatory" stage; the second is a hybrid EA.

- We do not use recombination operator.

- We use different mutation operator by the Virtual Loser method that was defined in [2]. In our operation the first part of the mutation operator makes random bit mutation, and the second part makes random bit mutation based on the Virtual Loser. The algorithm has maximum $\mathrm{n} / 5$ bit mutation in every generation in both parts of the mutation (it decreases a little bit during the evolutionary process). The number of the bit mutations was chosen based on the fitness landscape analysis in [1].

- We modified the update technique of the Virtual Loser method. We break periodically the update of the memory of the Virtual Loser and continue the evolution process with an empty memory.

- We speed up the convergence by a restart technique and by two special procedures. A Filter procedure selects only the best of the individuals close to each other, the other ones are deleted; and a Delete procedure deletes some worst individuals based on the fitness function periodically.

To improve the quality of the results and the running time we developed a parallel EA, an island model. In our island model:

- The basic EAs run on 2, 4, 8 or 16 parallel processors.

- The model uses a master-slave structure. It uses a centralized scheme in which slave processors execute the basic EA on their population and periodically send their best partial results to a master process. The master process stores the partial results in a common migration set $(M S)$, and chooses random individuals from MS one after the other for every slave and sends them to the slaves.

(With this island model we wanted to examine the performance of the island model's structure, so we simulated the island model in one processor). 
Table 1. Average solution values for large scale problems without the island model

\begin{tabular}{|c|c|c|c|c|c|c|c|c|c|c|c|c|}
\hline \multirow[t]{2}{*}{ Task } & \multicolumn{4}{|c|}{ Glov500 } & \multicolumn{4}{|c|}{ B1000 } & \multicolumn{4}{|c|}{ B2500 } \\
\hline & $\begin{array}{c}\text { Best } \\
\text { known }\end{array}$ & $A D$ & $\begin{array}{l}\text { AT/ } \\
\text { gen }\end{array}$ & $\begin{array}{l}\mathrm{b} / \\
20\end{array}$ & $\begin{array}{c}\text { Best } \\
\text { known }\end{array}$ & $A D$ & $\begin{array}{l}\text { AT/ } \\
\text { gen }\end{array}$ & $\begin{array}{l}\mathrm{b} / \\
20\end{array}$ & $\begin{array}{c}\text { Best } \\
\text { known }\end{array}$ & $A D$ & $\begin{array}{l}\text { AT/ } \\
\text { gen }\end{array}$ & $\begin{array}{l}\mathrm{b} / \\
20\end{array}$ \\
\hline 1 & 61194 & 0 & $9 / 51$ & 20 & 371438 & 0 & $133 / 113$ & 20 & 1515944 & 0 & $761 / 118$ & 20 \\
\hline 2 & 100161 & 0 & $10 / 48$ & 20 & 354932 & 0 & $73 / 76$ & 20 & 1471392 & 0.0053 & $2243 / 252$ & 12 \\
\hline 3 & 138135 & 0 & $17 / 62$ & 20 & 371236 & 0 & $53 / 58$ & 20 & 1414192 & 0 & $827 / 129$ & 20 \\
\hline 4 & 172771 & 0 & $30 / 97$ & 20 & 370675 & 0 & $75 / 83$ & 20 & 1507701 & 0 & $232 / 54$ & 20 \\
\hline 5 & 190507 & 0 & $23 / 75$ & 20 & 352760 & 0 & $106 / 98$ & 20 & 1491816 & 0 & $1071 / 155$ & 20 \\
\hline 6 & & & & & 359629 & 0 & $89 / 87$ & 20 & 1469162 & 0 & $1159 / 166$ & 20 \\
\hline 7 & & & & & 371193 & 0 & $159 / 126$ & 20 & 1479040 & 0 & $1832 / 237$ & 20 \\
\hline 8 & & & & & 351994 & 0 & $183 / 139$ & 20 & 1484199 & 0 & $1407 / 186$ & 20 \\
\hline 9 & & & & & 349337 & 0 & $223 / 168$ & 20 & 1482413 & 0 & $1394 / 229$ & 20 \\
\hline 10 & & & & & 351415 & 0 & $107 / 92$ & 20 & 1483355 & 0 & $1238 / 174$ & 20 \\
\hline Aver. & & 0 & $18 / 67$ & & & 0 & $120 / 104$ & & & 0.0005 & $1216 / 170$ & \\
\hline
\end{tabular}

\section{COMPUTATION EXPERIENCES}

We tested the basic EA with the benchmark set from the ORLibrary. We show only three benchmark sets (the large scale problems): two sets with 1000 and 2500 dimensions and the set with 500 dimensions (notation: B1000, B2500, glov500). In each set there are 5 or 10 instances. (The algorithm was implemented in Visual Basic and ran on a Pentium $41.8 \mathrm{GHz}$ with $256 \mathrm{MB}$ RAM).

Table 1 and table 2 present mean values calculated from a number of runs without and with parallel computing; all problems were run 20 times (The running was finished if the number of the generations was exceeded 400). In the tables we give the problem name (task), the average relative percentage deviation of the solution from the best known solution (AD) and the average running time in seconds (AT) with the average generation numbers (gen) to the best solutions. In table 1 we give how many best-known solutions could find the algorithm within the predefined number of generation $(b / 20)$.

We can say that our island model is successful; we could improve both the quality and the running times of the basic EA. The island model found the best-known solutions in all runs and the average running times are 2, 3 or 4 times shorter that on one processor without the island model.

We compared our results with one of the best method's results [1]. We can conclude that our model has similar results and it can find the best-known solutions with a high probability.

\section{ACKNOWLEDGEMENT}

The Hungarian Research Foundation OTKA K 68137 supported the study.
Table 2. Average solution values for large scale problems on 16 parallel processors

\begin{tabular}{|c|r|r|r|r|r|r|}
\hline \multirow{2}{*}{ Task } & \multicolumn{2}{|c|}{ Glov500 } & \multicolumn{2}{c|}{ B1000 } & \multicolumn{2}{c|}{ B2500 } \\
\cline { 2 - 7 } & AD & AT/gen & AD & AT/gen & AD & \multicolumn{1}{c|}{ AT/gen } \\
\hline 1 & 0 & $7 / 49$ & 0 & $26 / 49$ & 0 & $233 / 58$ \\
\hline 2 & 0 & $4 / 71$ & 0 & $22 / 44$ & 0 & $1012 / 132$ \\
\hline 3 & 0 & $6 / 47$ & 0 & $27 / 49$ & 0 & $295 / 64$ \\
\hline 4 & 0 & $7 / 48$ & 0 & $30 / 51$ & 0 & $136 / 47$ \\
\hline 5 & 0 & $8 / 47$ & 0 & $27 / 48$ & 0 & $173 / 50$ \\
\hline 6 & & & 0 & $26 / 50$ & 0 & $379 / 73$ \\
\hline 7 & & & 0 & $38 / 53$ & 0 & $574 / 96$ \\
\hline 8 & & & 0 & $40 / 64$ & 0 & $616 / 91$ \\
\hline 9 & & & 0 & $38 / 55$ & 0 & $374 / 73$ \\
\hline 10 & & & 0 & $37 / 55$ & 0 & $290 / 64$ \\
\hline Average & 0 & $6 / 46$ & 0 & $31 / 52$ & 0 & $408 / 75$ \\
\hline
\end{tabular}

\section{REFERENCES}

[1] Merz, P. and Katayama, K. A Hybrid Evolutionary Local Search Approach for the Unconstrained Binary Quadratic Programming Problem. Bio Systems. Vol. 78. No. 1-3.pp 99-118.

[2] Sebag, M., Schoenauer, M. and Ravisé, C. Toward Civilized Evolution: Developing Inhibitions. In: Bäck T (ed): Proc. of the $7^{\text {th }}$ International Conference on Genetic Algorithm. Morgan Kaufmann Pub. San Francisco, 1997. pp 291-298. 